\title{
BMJ Open Use of carnosine in the prevention of cardiometabolic risk factors in overweight and obese individuals: study protocol for a randomised, double-blind placebo-controlled trial
}

\author{
Kirthi Menon, ${ }^{1}$ James D Cameron, ${ }^{2,3}$ Maximilian de Courten, ${ }^{1,4}$ \\ Barbora de Courten (i) ${ }^{3}$
}

To cite: Menon K, Cameron JD, de Courten M, et al. Use of carnosine in the prevention of cardiometabolic risk factors in overweight and obese individuals: study protocol for a randomised, double-blind placebocontrolled trial. BMJ Open 2021;11:e043680. doi:10.1136/ bmjopen-2020-043680

- Prepublication history for this paper is available online. To view these files, please visit the journal online (http://dx.doi. org/10.1136/bmjopen-2020043680).

Received 12 September 2020 Revised 07 April 2021 Accepted 11 April 2021

\section{Check for updates}

(C) Author(s) (or their employer(s)) 2021. Re-use permitted under CC BY-NC. No commercial re-use. See rights and permissions. Published by BMJ.

For numbered affiliations see end of article.

\section{Correspondence to}

Professor Barbora de Courten; barbora.decourten@monash. edu

\section{ABSTRACT}

Introduction Carnosine, an over the counter food supplement, has been shown to improve glucose metabolism as well as cardiovascular risk factors in animal and human studies through its anti-inflammatory, antioxidative, antiglycating and chelating properties. The aim of this study is to establish if carnosine supplementation improves obesity, insulin sensitivity, insulin secretion, cardiovascular risk factors including arterial stiffness and endothelial function, and other risk factors related to diabetes and cardiovascular disease in the overweight and obese population.

Methods and analysis Fifty participants will be recruited to be enrolled in a double-blind randomised controlled trial. Eligible participants with a body mass index (BMI) between 25 and $40 \mathrm{~kg} / \mathrm{m}^{2}$ will be randomly assigned to the intervention or placebo group. Following a medical review and oral glucose tolerance test to check eligibility, participants will then undergo testing. At baseline, participants will have anthropometric measurements (BMI, dual X-ray absorptiometry and peripheral quantitative CT scan), measurements of glucose metabolism (oral glucose tolerance test, intravenous glucose tolerance test and euglycaemic hyperinsulinaemic clamp), cardiovascular measurements (central blood pressure, endothelial function and arterial stiffness), a muscle and fat biopsy, physical activity measurement, liver fibroscan, cognitive function and questionnaires to assess dietary habits, sleep quality, depression, and quality of life. Following baseline assessments, participants will be randomised to either $2 \mathrm{~g}$ carnosine or placebo for 15 weeks. In the 15th week, all assessments will be repeated. The preplanned outcome metric is the change between baseline and follow-up measures.

Ethics and dissemination This study is approved by the Human Research Ethics Committee of Monash Health and Monash University, Australia.

Trial registration number NCT02686996.

\section{INTRODUCTION}

Obesity, an important cause of premature mortality, represents one of the biggest public health concerns faced today. The

\section{Strengths and limitations of this study}

- Employs gold-standard methodology to measure insulin sensitivity and comprehensively assesses cardiometabolic risk factors in an overweight and obese population.

- Inclusion of only overweight and obese individuals means that results may not be generalisable to other populations.

- Secondary outcomes may be insufficiently powered to detect a difference due to a small sample size.

global prevalence of obesity has tripled since 1975 making its management a global health challenge. ${ }^{1}$ Globally $13 \%$ of adults are obese. ${ }^{1}$ Obesity increases the risk of type 2 diabetes mellitus (T2DM), cardiovascular disease (CVD), several cancers, osteoarthritis and liver disease, ${ }^{23}$ making it imperative to control this epidemic.

The underlying cause of obesity is multifactorial, a combination of genetic, environmental, behavioural and social factors. ${ }^{4} 5$ The epidemic is further fuelled by a shift to primarily high caloric meals and sedentary lifestyles. The management of obesity encompasses one or a combination of lifestyle interventions, pharmacotherapy and surgery, with lifestyle often being first line but also an ongoing strategy. Lifestyle interventions, however, can be difficult to maintain in the long run and pharmacotherapy is not without side effects. Strategies that enhance lifestyle intervention without the burden of side effects are rare but highly desirable.

Inflammation, oxidative stress and advanced glycation are important features in the pathogenesis of many chronic diseases including diabetes and CVD and, are worsened by obesity. ${ }^{6}$ The ideal intervention for 
the management of chronic diseases should target the underlying mechanisms of the disease.

Carnosine is a naturally available dipeptide (betaalanine histidine) and is naturally present in meat and fish $^{78}$ but cooking practices can alter the amount available making an over the counter supplement a more practical source of carnosine. It has potent anti-inflammatory, anti-glycating, antioxidative and chelating ${ }^{9-12}$ properties which suggest the potential for prevention of chronic diseases. Several animal and human studies have examined the role of carnosine in the prevention of chronic diseases including obesity, T2DM, CVD, neurodegenerative diseases and other chronic diseases linked to inflammation, oxidative stress and advanced glycation.

Animal studies have reported that carnosine supplementation reduced plasma glucose, improves insulin sensitivity, preserves insulin secretion, delays the development of type 2 diabetes and improves markers of inflammation, oxidative stress and advanced glycation. ${ }^{10}$ 13-18 Carnosine has also been shown to improve lipid profile and inhibit the development of atherosclerosis ${ }^{15} 19$ and improve cognitive function. ${ }^{20}$ In a pilot trial, we have recently shown improvements in glucose metabolism in overweight and obese adults following 12 weeks of carnosine supplementation $(2 \mathrm{~g} /$ day $)$ with the largest effect in those with pre-diabetes. ${ }^{21}$ Other benefits include improvements in fat mass and fat-free mass. ${ }^{22}{ }^{23}$ Our two recent meta-analysis showed improvements in fasting glucose, haemoglobin A1c (HbA1c) and triglyceride levels in studies using carnosine as a part of the intervention. ${ }^{2425}$
Moreover, other human studies using carnosine reported improved quality of life, physical performance and cognitive function. ${ }^{202627}$ Despite several studies showing the beneficial effects of carnosine on cardiometabolic risk factors, several limitations exist in the current literature. Several studies used mixed supplements including varying doses and duration of treatment. There is yet to be a study using gold-standard measures of glucose metabolism and recommended cardiovascular measures.

To further our understanding of the role of carnosine in the prevention of diabetes and cardiovascular risk factors and address current limitations and knowledge gaps, we aim to perform a randomised placebo-controlled trial in overweight and obese individuals to examine the effect of carnosine supplementation on obesity, insulin sensitivity, insulin secretion and cardiovascular risk factors including arterial stiffness, endothelial function and other risk factors related to these conditions and to study the mechanisms involved.

\section{METHODS AND ANALYSIS}

\section{Study design and setting}

This study is a parallel arm double-blind randomised placebo-controlled trial (figure 1). We have adhered to the Standardised Protocol Interventions: Recommendations for Interventional Trials 2013 Statement which defines standard protocol items for clinical trials. ${ }^{28}$ Fifty overweight or obese participants (body mass index, BMI $\geq 25$ to $\leq 40 \mathrm{~kg} / \mathrm{m}^{2}$ ) aged between 18 and 70 years of age

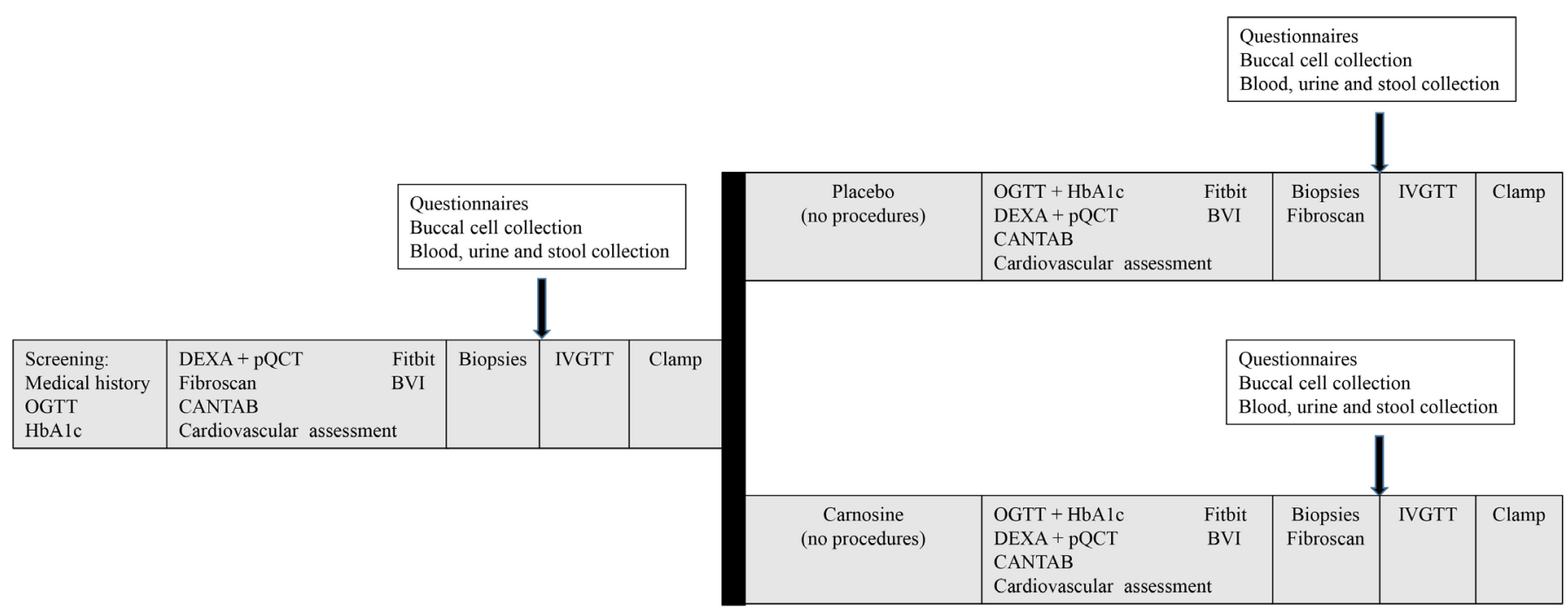

Day -

Day 1-4

Day 1-4

Week - 1

Week 1

Week 1-14

Week 15

SCREENING

RANDOMISATION

FOLLOW-UP

Figure 1 Study timeline. BVI, body volume indicator; CANTAB, Cambridge neuropsychological test automated battery; DEXA, dual energy X-ray absorptiometry; HbA1c, haemoglobin A1c; IVGTT, intravenous glucose tolerance test; OGTT, oral glucose tolerance test; pQCT, peripheral quantitative CT scan. 
with no other medical problems will be included in the study. Participants will be randomised to either receive $2 \mathrm{~g}$ of carnosine or a matching placebo in two divided doses for 15 weeks. The 15-week duration was chosen based on a carnosine pilot trial led by the chief investigator of the trial. ${ }^{21}$ Potential participants will be recruited through several advertising platforms including posters, newspapers, radio announcements, email newsletters of Monash University and Monash Health, social media and community websites. Participation will be voluntary, and participants can withdraw at any time.

\section{Inclusion and exclusion criteria}

Non-smoking individuals aged between 18 and 70 years with a BMI between 25 and $40 \mathrm{~kg} / \mathrm{m}^{2}$ and stable body weight (no weight gain or loss $>5 \mathrm{~kg}$ in the 6 months preceding the trial) will be eligible for the study. Individuals with morbid obesity (BMI $>40 \mathrm{~kg} / \mathrm{m}^{2}$ ), any renal, cardiovascular, haematological, respiratory, gastrointestinal, endocrine, central nervous system diseases, psychiatric disorders or active cancer in the preceding 5 years or the presence of acute inflammation based on the medical history, physical examination and laboratory results will be excluded. Individuals who donated blood or have a history of blood transfusions in the preceding 3 months, on any medications, supplements or vitamins, are current smokers will be also excluded or deferred until they comply with the study inclusion and exclusion criteria and washout has been ensured. Pregnant and lactating women will also be excluded.

\section{Sample size calculation}

Based on the data from a similar cohort of overweight and obese participants in our laboratory, which has an insulin sensitivity measured by glucose clamp of $8.1 \pm 2.0$ (mean $\pm \mathrm{SD}$ ) (unpublished data), a sample size of 22 completing each arm will be required to detect a $20 \%$ change in insulin sensitivity between carnosine supplement and control arm. We selected a $20 \%$ difference because treatment with an insulin sensitiser, troglitazone, in patients with obesity resulted in similar improvements in insulin sensitivity (measured by clamps) ${ }^{29}$ and a reduction in the incidence of type 2 diabetes. ${ }^{30}$ An additional six participants in each arm $(15 \%)$ will be included to allow for non-completion. Based on a type I error of 0.05 (two tail) and a power of $80 \%$, we will need a total of 50 participants recruited to complete the study.

\section{Screening}

At the first visit, participants sign the consent form (online supplemental file 1) followed by a medical review which includes a history and physical examination (including vital signs and anthropometry) by a registered medical practitioner involved in the study. Screening bloods will be taken for full blood count, kidney and liver function, calcium, magnesium and phosphate levels, lipid profile, $\mathrm{HbA1c}$, and $\mathrm{C}$ reactive protein (CRP) and will be sent to Monash Health Hospital Pathology for analysis.
Participants will then undergo a $75 \mathrm{~g}$ oral glucose tolerance test (OGTT).

\section{Baseline assessments}

Eligible participants will commence the remainder of the baseline assessments at the next visit. The second visit will involve assessment of whole-body composition (using dual X-ray absorptiometry), measurement of muscle and fat tissue density of the skeletal muscle in the non-dominant leg (peripheral quantitative CT, pQCT), physical activity (Fitbit), measurement of endothelial function (Endo PAT), central blood pressure and arterial stiffness assessed by carotid-femoral pulse wave velocity (PWV) (Complior), liver fibroscan, body volume indicator (BVI) and a cognitive assessment using the Cambridge Neuropsychological Test Automated Battery for Prodromal Alzheimer's Disease (CANTAB). The third visit will involve a muscle biopsy from the vastus lateralis and adipose tissue from the subcutaneous abdomen. The fourth visit will involve an intravenous glucose tolerance test (IVGTT) and the final baseline visit will include a euglycaemic hyperinsulinaemic clamp. Blood samples will be collected for measurement of markers of inflammation (including interleukin (IL)-1 $\beta$, IL-6, IL-8 and IL-10, and CRP), carnosine levels, advanced glycation end products (AGEs), advanced lipid oxidation end products (ALEs) and DNA damage tests. Participants will also be required to complete questionnaires assessing a range of areas including dietary habits, quality of life, presence of depression, quality of life, musculoskeletal pain and physical activity. In addition, a urine sample will be collected for measurement of urine albumin creatinine ratio, urine carnosine levels, and AGE/ALE levels and stool sample for measurement of microbiome and buccal samples for measurement of DNA.

\section{Randomisation}

Following the completion of successful screening and baseline measures, participants will be randomised into carnosine and placebo groups in block sizes of 4 by sex to ensure balance between the sexes in each group. The active group will receive $2 \mathrm{~g}$ of carnosine (two times daily) for 15 weeks while the placebo group will receive methylcellulose for 15 weeks. Both carnosine and placebo capsules will be identical to ensure both participants and investigators (including outcome assessors and data analysts) are blinded to the treatment. The randomisation codes generated by the study statistician will be sent to the Monash Health Clinical Trial Pharmacy for dispensing. To maximise compliance, all participants will be contacted at 6 weeks. Participants are encouraged to return all medication bottles at the end of the trial for a compliance check.

\section{Intervention}

Following randomisation, participants will be given four capsules of carnosine (Flamma S.p.A, Italy) or matching capsules of placebo to be taken daily (two capsules of 
$500 \mathrm{mg}$ two times daily), a total of 420 capsules for the duration of the study. The dose of $2 \mathrm{~g}$ daily was selected based on our pilot intervention study ${ }^{21}$ which demonstrated a change in glucose metabolism. All participants will be instructed to maintain their usual daily activities and make no change to their dietary habits.

\section{Follow-up visits}

Participants are scheduled for follow-up in the final week of their supplementation. All baseline measures are repeated at follow-up including screening bloods (full blood count, kidney and liver function, calcium, magnesium and phosphate levels, lipid profile, HbAlc and CRP), blood pressure measurement, anthropometry, OGTT, central blood pressure, endothelial function and arterial stiffness, BVI, liver fibroscan, percutaneous muscle and adipose tissue biopsies, IVGTT and glucose clamp. All participants will also be asked to complete the same set of questionnaires as completed at baseline.

\section{Safety considerations}

During the screening, baseline and follow-up visits, any medical condition or laboratory abnormalities will be promptly discussed with the participants by a qualified medical practitioner involved in the study. Anything that requires urgent medical attention will be discussed with the participant and a referral to the participant's general practitioner or other appropriate practitioners will be made. Non-urgent results will be discussed with the participants after completion of the trial. Participants will also be given results of any clinically relevant blood tests and advice about improving their diabetes and cardiovascular risk factors at the end of the trial. All adverse events will be recorded and reported to Monash University and Monash Health Human Research Ethics committees. All major adverse events will be reported to the safety monitor who may choose to unblind randomisation in response to an adverse event.

\section{Outcome assessments}

The primary outcome of this study is the difference in insulin sensitivity (measured by euglycaemic glucose clamp) between the carnosine and placebo groups. Secondary outcomes include changes in body fat, insulin secretion, systolic and diastolic pressures, lipid profile (total cholesterol, triglycerides, low-density lipoprotein cholesterol (LDL-C) and high-density lipoprotein cholesterol (HDL-C), endothelial function and arterial stiffness. Tertiary outcomes liver fibroscan, waist-to-hip ratio, sleep quality, kidney function, inflammatory markers including IL-1 $\beta$, IL-6, IL-8 and IL-10, CRP, tumour necrosis factor $\alpha(\mathrm{TNF} \alpha)$, macrophage migration inhibitory factor (MMIF), monocyte chemoattractant protein-1 (MCP$1)$, nuclear factor kappa beta (NF- $\kappa \beta)$ activity, AGEs and ALEs. Gene expression of inflammatory markers will be analysed in muscle and adipose tissue samples. Additionally, other measures such as insulin signalling proteins will be measured in these tissues. Self-reported questionnaires will also be assessed.

\section{ETHICS AND DISSEMINATION}

This trial has received ethics approval from Monash Health (ID number: 16061A) and Monash University (ID number: 7787), Melbourne, Australia. Any amendment to the trial protocol will be communicated to the ethics committees. The trial commenced in February 2017 and is still recruiting participants. Prior to the commencement of any study procedure, participants will provide written informed consent. Study findings will be presented at national and international scientific meetings and published in peer-reviewed journals.

\section{Data management}

Databases created as part of this project will include identifiable participant information and deidentified research outcome data. Deidentified data will be reidentifiable by a participant code, linked to identifiable participant information.

Electronic data will be stored within a passwordprotected database within the Monash University information technology network and further protected by the Monash firewall and other network-related security measures. Only investigators with prior ethics approval will have access to this data. Additionally, hard copies of any participant identifying information will be stored in a locked cabinet in a secure facility of Monash University. All biological samples will be collected following recommended lab practices.

\section{Statistical analysis}

Data analysis will be conducted according to the intentionto-treat principle. All outcomes will be reported as change from baseline and this change will be compared between the treatment groups. The effects of the intervention on the outcomes (between-group differences) will be analysed using independent student $t$-tests and linear regression model to account for covariates such as age, sex, diet, physical activity and carnosinase activity if necessary. The assumptions for the tests will be assessed and data transformation and non-parametric tests applied when appropriate. Categorical variables will be analysed using the $\chi^{2}$ test for equal proportion. A formal interim analysis is planned when 28 patients have been recruited and have the endpoints successfully assessed. The trial will be stopped early for efficacy when the t-statistic crosses the 2.9626 level for the primary endpoint insulin sensitivity, according to the sequential O'Brien-Fleming alpha spending function proposed by Lan and DeMets. ${ }^{31} \mathrm{P}$ values of $<0.05$ will be considered statistically significant, with the $\mathrm{p}$ value adjusted for multiple comparisons (11 secondary outcomes) where relevant, except for the primary endpoint where the level of significance will be set at 0.047 since there is an interim analysis performed. Subgroup analyses by gender and BMI will also be 
assessed. Data analysis will be performed using Stata V.14.0 (StataCorp).

\section{DATA COLLECTION AND ANALYSIS \\ Anthropometry \\ Body mass index}

Body weight is measured using a digital scale (Tanita BWB-600) while height is measured using a stable stadiometer (Seca 206, Australia) at baseline and follow-up. BMI will be calculated using the formula weight $(\mathrm{kg}) /$ height $\left(\mathrm{m}^{2}\right)$. Body weight measures will be taken without shoes.

\section{Waist to hip ratio}

Waist and hip circumference will first be measured to calculate the ratio (waist $(\mathrm{cm}) / \mathrm{hip}(\mathrm{cm})$ ) using a constant-tension tape. Waist circumference is measured at the midpoint between the upper iliac crest and the lowermost rib at the end of expiration and hip circumference is measured at the widest part of the buttocks.

\section{Body fat composition}

Whole body fat composition will be measured noninvasively using a dual energy X-ray absorptiometry. It allows measurement of total body composition including fat tissue mass, lean tissue mass and bone mineral content. It is a safe, accurate and validated tool for measuring soft tissue body composition by region. ${ }^{32}$

\section{Body volume indicator}

BVI is a new mobile measurement tool for obesity (BVI, Select Research). It uses 3D Body Volume Imaging technology to measure total body fat, visceral fat, waist-to-hip ratio, abdomen volume and BMI as well as BVI through the BVI Pro app installed on the iPad.

\section{Muscle and fat tissue density in skeletal muscle}

Muscle and fat tissue density of the non-dominant leg will be measured using pQCT (Stratec XCT3000, Stratec, Germany). pQCT provides three dimensional analysis of trabecular and cortical bone components and also measures several bone geometrical parameters in addition to bone mineral density. ${ }^{33}$ The cross-sectional area of muscle and fat can also be extracted using this measure.

\section{Metabolic studies}

Oral glucose tolerance test

After an overnight fast (10-12hours), participants will ingest $75 \mathrm{~g}$ of oral glucose over $2 \mathrm{~min}$ and have serum glucose and insulin levels measured at $30 \mathrm{~min}$ intervals $(0,30,60,90$ and $120 \mathrm{~min})$.

\section{Intravenous glucose tolerance tests}

Acute insulin secretory response is measured in response to a $25 \mathrm{~g}$ load of intravenous glucose $(125 \mathrm{~mL}$ of $20.8 \%$ dextrose administered over $3 \mathrm{~min}$ and $27 \mathrm{~s}$ ). Following an overnight fast, baseline bloods are collected at -10 and $0 \mathrm{~min}$ after which $25 \mathrm{~g}$ of intravenous dextrose is administered, bloods are collected at 3, 4, 6, 8, 10, 15, 20, 25 and $30 \mathrm{~min}$ to measure glucose and insulin levels.

\section{Hyperinsulinimic euglycaemic clamp}

Insulin sensitivity is measured using the hyperinsulinimic euglycaemic clamp. After a set of baseline bloods are taken to measure glucose and insulin levels, an intravenous bolus of insulin $(9 \mathrm{mU} / \mathrm{kg})$ is given followed by a constant insulin infusion rate of $40 \mathrm{mU} / \mathrm{m}^{2}$ for approximately $120 \mathrm{~min}$ along with an intravenous dextrose infusion at a varying rate to maintain euglycaemia. Plasma glucose will be measured every $5 \mathrm{~min}$ and the glucose infusion rate will be adjusted to maintain glucose levels at $5 \mathrm{mmol} / \mathrm{L}$.

\section{Percutaneous muscle and fat biopsy}

A muscle biopsy will be obtained from the vastus lateralis (approximately $10 \mathrm{mg}$ of muscle sample) under local anaesthetic and the fat biopsy (approximately $10 \mathrm{~g}$ of fat sample) from the abdomen. Samples will be immediately frozen and stored at $-80^{\circ} \mathrm{C}$ for analysis at a later stage.

\section{Cognitive}

Cognitive function is measured using several different platforms including CANTAB for Prodromal Alzheimer's disease (CANTAB software, UK) ${ }^{34}$ Victoria Stroop, Trail making and Digit Symbol Substitution.

\section{Liver fibroscan}

Non-invasive transient elastography (Fibroscan, EchoSens, France) will be used to measure liver fibrosis by measurements of liver fat and stiffness. ${ }^{35}$ An ultrasound transducer probe is used to determine the speed of the wave emitted from the probe towards the liver. The velocity of the wave is proportional to the density, stiffness or degree of fibrosis in the liver. The properties of the ultrasound signals also measure the degree of liver fat.

\section{Cardiovascular studies}

\section{Blood pressure}

A resting systolic and diastolic blood pressure will be measured three times using an automated oscillometric system (Omron, Australia). The average blood pressure of the three readings will be recorded.

\section{Lipid profile}

Lipid profile parameters to be measured include total cholesterol, triglycerides, LDL-C and HDL-C using a standard commercial enzymatic assay, a Beckman Coulter LX20PRO analyser and SYNCHRON Systems lipid and multi calibrators (Beckman Coulter Diagnostics, Australia). Besides lipid profiles, a lipidomic analysis will also be undertaken. ${ }^{36}$

\section{Arterial stiffness and central pressure}

Aortic (carotid-femoral) PWV (aPWV) is measured using the Complior device (Alam Medical, France). Pulse transit time-the time between successive arrivals of the foot of simultaneously recorded pressure waves-is averaged over 
10 cycles and velocity is derived from $\mathrm{PWV}=\mathrm{D} / \Delta \mathrm{t}(\mathrm{m} / \mathrm{s})$, where (D) distance is measured in accordance with current guidelines of the European Working Group on LARGE Arteries. ${ }^{37} 38$ Central blood pressure and aortic augmentation index will also be assessed by this device.

\section{Endothelial function}

Continuous plethysmographic signals of the finger arterial pulse wave is recorded using non-invasive peripheral arterial tomography ${ }^{39}$ (EndoPat, Itamar Medical, Israel). Finger probes are placed on each index finger and after 5 min of equilibration, a blood pressure cuff in the nondominant arm is inflated to $200 \mathrm{~mm} \mathrm{Hg}$ to occlude blood flow for $5 \mathrm{~min}$. After a $5 \mathrm{~min}$ occlusion period, the cuff is deflated to induce reactive hyperaemia. Postocclusion changes are then monitored for $10 \mathrm{~min}$. Results are normalised to the non-occluded arm, compensating for potential systemic changes (RH-PAT).

\section{Physical function and strength}

Physical activity

Physical activity will be measured using a Fitbit Charge Heart rate (Fitbit, USA) which participants will wear for five consecutive days. In addition, participants will complete a physical activity diary for the 5-day period. Data collected from the Fitbit includes the number of steps per day, average heart rate and minutes of moderate or vigorous activity. The screen on the Fitbit will be covered to ensure participants are not influenced by the data collected.

\section{Handgrip strength}

Handgrip strength is measured in both hands using the Jamar hydraulic handgrip dynamometer. Participants will hold the dynamometer with their elbow at $90^{\circ}$ while the other hand is resting on their lap. The hand holding the dynamometer will apply as much force as possible for three seconds. The test is repeated three times with a $30 \mathrm{~s}$ gap between tests and the mean of the last two readings recorded as the measure for handgrip strength.

\section{Knee extension strength}

Knee extension strength is measured in the dominant leg using Baseline Cable Tensiometer (Fabrication Enterprises, USA). The test is done with participants sitting upright in a stool and with their hands resting on their laps. The tensiometer is secured to the rear leg of the stool which corresponds to the leg that is to be tested. A Velcro strap is secured to the participant's leg and maximal force is applied to move the leg forward for three seconds. The peak force achieved in kilograms is indicated on the tensiometer dial. The test is performed three times with the mean score for each leg taken as the value for quadriceps strength.

\section{Laboratory measurements}

Measurement of carnosine and carnosinase

Serum and urine carnosine levels will be analysed using high-performance liquid chromatography/electrospray ionisation tandem mass spectrometry (ESI-MS) (triple quadruple and orbitrap MS analyser). Metabolites of carnosine from covalent detoxification of the reactive carbonyl species (RCS) (precursors of AGEs and ALEs) will be analysed similarly. Plasma carnosinase protein will be measured by ELISA for human carnosinase one with a monoclonal antibody (clone ATLAS, Abcam) and peroxidase substrate. ${ }^{40}$

\section{Measurement of AGES and ALES}

Plasma and urinary AGEs and ALEs will be measured using liquid chromatography-tandem MS and ELISA tests. ${ }^{41}$ Circulating receptors for AGEs will be measured using ELISA. ${ }^{42}$ Protein modification and the effect of carnosine supplementation will be determined by proteomic approaches. Systemic oxidative status will be determined by measuring the advanced oxidation protein products and by measuring the cysteinylated form of albumin by MS. Mercapturic acid adducts with the main RCS will also be quantitatively determined by LC-ESI-MS $/ \mathrm{MS}^{41}$

\section{Inflammatory markers}

Plasma inflammatory markers (IL-1 $\beta$, IL-6, IL-8 and IL-10, TNF $\alpha$, MMIF and MCP-1) will be measured using a bead based, multiplex assay (multianalyte flow assay kit, LEGEND Plex, BioLegend, CA, USA), while plasma CRP will be analysed via highly sensitive near infrared particle immunoassay rate methodology and a Beckman Coulter Synchron LX system chemistry analyser (Beckman Coulter, Australia).

$\mathrm{NF}-\kappa \beta$ activity

Nuclear extracts of white bloods cells will be obtained and analysed for the binding capacity of the p50/p65 subunit of NK- $\kappa \beta$ to an NK- $\kappa \beta$ oligonucleotide consensus sequence as per the manufacturer's instructions (Active Motif, California, USA)

\section{DNA damage tests}

Telomere shortening and mitochondrial DNA deletions will be measured in frozen isolated lymphocytes and buccal cells. These will be done according to a previously published protocol. ${ }^{434}$ Buccal cells will be collected from the check cells inside the mouth using a soft toothbrush.

\section{Self-reported questionnaires}

Quality of life

The EuroQol 5 dimensions questionnaire is a widely used instrument to measure health-related quality of life. There are five dimensions of the questionnaire which are mobility, self-care, usual activities, pain/discomfort and anxiety/depression. ${ }^{45}$ Each dimension has three levels which are no problems, some problems, and extreme problems (labelled as 1-3). Participants rate each domain based on what is best suited to their health state.

\section{Depression scale}

The Personal Health Questionnaire Depression Scale is a validated self-reported eight item questionnaire used as a 
measure to assess participants' depression over the last 2 weeks. ${ }^{46}$ Participants score each item from 0 (not at all) to 3 (nearly every day).

\section{International Physical Activity Questionnaire}

The short version of the International Physical Activity Questionnaire will be used to monitor participant physical activity over the past 7 days. The questionnaire asks participants how much time they spent doing vigorous activity, moderate activity, walking and sitting. ${ }^{47}$

\section{Sleep quality}

The Pittsburgh Sleep Quality Index is used to assess the sleep habits of participants over the preceding month. ${ }^{48}$ In addition, participants will also complete the Pittsburgh Sleep Diary and Stanford Sleepiness Scale for 14 consecutive days which will assess the usual sleeping habits of participants. ${ }^{490}$

\section{Record of habitual diet}

The Food Preference Questionnaire determines the participants' preference for high-fat/low-fat foods in 72 various food items. Each item is rated across a 9-point Likert scale which goes from 1- dislike extremely to 5-neutral and 9-extremely likely. The fat preference score will be a ratio of high-fat to low-fat score. Participants will also complete a food diary on three consecutive days (one of the 3 days being a weekend) to assess dietary intake (energy, macronutrient, micronutrient, food groups). Data from the food diaries will be analysed using the Foodworks 7 Professional Dietary Software (Xyris Software, Australia).

\section{Patient and public involvement}

Neither patients nor the public was involved in the design, or conduct, or reporting, or dissemination plans of this research.

\section{DISCUSSION}

This randomised controlled trial will examine the effect of carnosine supplementation on cardiometabolic risk factors and cognitive function and further our understanding of the mechanisms of action of carnosine in overweight and obese individuals.

The literature on the role of carnosine in obesity has been inconsistent. While there have been some animal studies that have reported an effect on body weight, ${ }^{15} 51$ others have not. ${ }^{52}$ Similarly, human studies show mixed effects on measures of adiposity, ${ }^{21}{ }^{22}$ however, only a few examined the effect in obese individuals. ${ }^{21}$ Only one study reported a significant reduction in fat mass and an increase in fat-free mass after 12 weeks of carnosine supplementation. ${ }^{22}$ In a recent meta-analysis, we reported a reduction in waist circumference in the intervention arm using histidine-containing dipeptides. ${ }^{25}$

Carnosine has been studied extensively in animal studies and has exhibited the potential to improve glycaemic measures. Forsberg et $_{\mathrm{al}} \mathrm{l}^{4}$ showed a decrease in blood glucose and an increase in insulin in the carnosine supplemented group of $\mathrm{db} / \mathrm{db}$ mice compared with control. In non-diabetic mice on a high-fat saturated diet, there was an improvement in insulin sensitivity following 8 weeks of carnosine supplementation. ${ }^{15}$ In a study involving BTBR ob/ob mice (an obesity-related diabetes model), 18 weeks of carnosine supplementation reduced plasma glucose and $\mathrm{HbA1c}$ and raised insulin and C-peptide levels. ${ }^{53}$ One study has even shown carnosine to delay the development of T2DM. ${ }^{16}$ Besides glycaemic measures, carnosine has been shown to attenuate hepatic steatosis. ${ }^{15}$ Human studies confirm animal data that carnosine has a similar effect on glycaemic measures. We have previously reported in a pilot study an improvement in glucose metabolism after carnosine treatment. ${ }^{21}$ Other studies that have shown an improvement in glycaemic measures and markers of insulin resistance in high-risk population such as obesity and type 2 diabetes. A study by Houjeghani et al demonstrated a reduction in fasting glucose and HbA1c levels after 12 weeks of carnosine supplementation. ${ }^{22}$ We have recently collated all studies in a metaanalysis and reported a $0.76 \%$ improvement in HbA1c and $0.5 \mathrm{mmol} / \mathrm{L}$ reduction in fasting glucose in the intervention arm using carnosine as a part of the intervention compared with control. ${ }^{25}$ Importantly, no study in the meta-analysis used IVGTT and glucose clamps which will be utilised in this study.

Besides glycaemic measures, the effect of carnosine on cardiovascular risk factors such as lipids, blood pressure, renal function and diseases has been explored in animal studies. Cholesterol and triglyceride levels in the heart and liver tissues of diabetic Balb/cA mice ${ }^{10}$ were reduced following carnosine supplementation. In apolipoprotein E-null mice, carnosine prevented atherogenesis by the removal of lipid peroxidation derived aldehydes. ${ }^{54} \mathrm{~A}$ study by Aldini et $a \tilde{l}^{\tilde{1}}$ in obese Zucker rats demonstrated a reduction in both lipid (cholesterol and triglycerides) and blood pressure measures in the carnosine intervention group. Similarly, lipid levels (cholesterol) improved in pigs supplemented with carnosine. ${ }^{55}$ Carnosine was also noted to have renoprotective effects in this study and protected the rats against the development of albuminuria and proteinuria. Furthermore, there are several animal studies that have shown that carnosine protects against cerebral ischaemia ${ }^{56}$ and can reduce the size of ischaemic lesions in several tissues including the heart, kidney, liver and above-mentioned brain. ${ }^{57-59}$ Human studies have also demonstrated a reduction in lipid levels specifically triglyceride and LDL-cholesterol levels and plasma lipidome. ${ }^{22} 3660$ In a group of patients with type 1 diabetes and diabetic nephropathy, 12 weeks of carnosine supplementation reduced urine albumin creatinine ratios, again highlighting a possible renoprotective component, along with improvements seen in glycaemic (HbA1c) and lipid measures (total cholesterol, triglycerides and HDL-cholesterol) ${ }^{61}$ Our meta-analysis showed a reduction in total cholesterol and triglyceride levels in the intervention arm (studies using carnosine/ 
its precursors and other histidine-containing dipeptides) compared with the control group. Our study will help establish the effects of carnosine supplementation in improving cardiovascular risk factors in a targeted population at risk of developing T2DM and CVD. Additionally, unlike many studies that have not included other supplements along with carnosine in the intervention, our study uses carnosine alone.

Carnosine supplementation has been also shown to have a beneficial role in Alzheimer's dementia and ischaemic vascular dementia. ${ }^{20}$ Rodent studies have shown that carnosine supplementation reduced in amyloid accumulation in the hippocampus preventing Alzheimer's dementia ${ }^{62}$ and white matter lesions and subcortical ischaemic lesions in vascular dementia both of which improved cognition. ${ }^{63}$ Several human studies have also shown the benefit of carnosine for $\operatorname{cognition}{ }^{20}$ Carnosine supplementation improved cognitive performance in young healthy soldiers and elderly individuals in two separate studies. ${ }^{27} 64$ Patients with Parkinson's disease showed an improvement in their neurological symptoms with carnosine supplementation. ${ }^{65}$ Contrary to this, there is also literature to suggest that beta-alanine (precursor of carnosine) has had no influence on cognition in a group of trained cyclists. ${ }^{66}$ In this randomised controlled trial, we will examine the impact of carnosine on various aspects of cognitive function in overweight and obese people since obesity is linked to cognitive dysfunction through a number of postulated mechanisms including vascular dysfunction and insulin resistance although the exact mechanism is yet to be fully understood ${ }^{67}$ Although carnosine is seen as a promising therapy for some of these neurological and psychiatric conditions, the limitation of current evidence includes its short study duration and the use of mixed supplements containing carnosine.

A number of mechanisms have been postulated to explain the effects of carnosine on glycaemic measures. First, carnosine has been shown to increase insulin and C-peptide levels and beta-cell mass ${ }^{14} 1653$ as well as improve insulin signalling ${ }^{68}$ which may explain improvements observed in glycaemic measures. Carnosine may also have an effect on glucose metabolism through its effect on glycolysis, ${ }^{69}$ a pathway that regulates insulin secretion. ${ }^{70}$ Additionally, the anti-inflammatory and antiglycating properties of carnosine minimise the harmful effects of inflammation, oxidative stress and glycation end-products which have the potential to impair insulin sensitivity, beta-cell function and cause beta-cell death. ${ }^{712}$

With regard to observed with lipid measures, carnosine has been shown to reduce the activity of enzymes involved in lipid metabolisms such as malic enzyme, fatty acid synthase, and 3-hydroxy-3-methylglutaryl coenzyme-A (HMG-CoA) reductase, subsequently lowering cholesterol and triglycerides levels. ${ }^{15}$ Carnosine is also believed to suppress sterol regulatory element-binding proteins which regulate fatty acid synthase and HMG-CoA reductase. ${ }^{15}$ Reduced expression of fatty acid synthase and HMG-CoA reductase lowers triglyceride and cholesterol levels in tissues. This is the same mechanism that is believed to cause weight loss in carnosine supplemented groups. Improvements in blood pressure as was evident in one rodent study following carnosine supplementation is believed to be due to carnosine's RCS quenching properties since RCS causes vascular wall thickening and loss of elasticity leading to hypertension. ${ }^{51}$ Additionally, carnosine has suppressive effects on the sympathetic system, ${ }^{11}$ another mechanism by which may exert its antihypertensive effects.

Several suggested mechanisms can explain improvements in cognition observed in animal and human studies. One study has postulated that daily carnosine could restore memory deficits in an Alzheimer's mouse model through lowering the expression of receptors of AGE in blood vessels and inhibiting microglial activation in the hippocampus. ${ }^{73}$

While many studies have demonstrated the benefits of carnosine, it is important to note some of the gaps in the literature. These include the heterogeneity of the populations in the studies, the different doses and durations of carnosine supplementation, and carnosine studied as a part of mixed supplements, and the lack of gold-standard methodology to measure these outcomes most of which we will address in this study. To understand and establish if carnosine can improve glycaemic, cardiovascular, and cognitive outcomes in the overweight and obese population particularly since this chronic condition has reached alarming levels, a clinical trial designed to address these knowledge gaps is essential. If carnosine proves to be efficacious, it can be a low-cost recommended treatment option in the management of chronic conditions like obesity, T2DM and CVD.

Obesity and its consequences such as T2DM, CVD, neurodegenerative diseases as well as depression and anxiety are at unprecedented levels becoming a burden to health and the economy. This emphasises the need for a safe, cheap, scalable and effective intervention to help change the trajectory of this escalating burden of disease. Many animal and human studies have already demonstrated a benefit of carnosine in the prevention and management of chronic diseases, however, there is a lack of evidence in using gold-standard methodologies such for assessment of glucose metabolism. If we demonstrate an improvement in insulin sensitivity from this study, there is a potential for carnosine to be used in highrisk cohorts, that is, patients with obesity, pre-diabetes, and T2DM to improve risk factor profile and glycaemic control, respectively. There is therefore a need for a welldesigned clinical trial investigating the role of carnosine in cardiometabolic health, cognition and depression/ anxiety in individuals with obesity.

\section{Author affiliations}

${ }^{1}$ School of Public Health and Preventive Medicine, Monash University, Melbourne, Victoria, Australia

${ }^{2}$ MonashHeart and Monash Cardiovascular Research Centre, Melbourne, Victoria, Australia

${ }^{3}$ School of Clinical Sciences, Monash University, Melbourne, Victoria, Australia 
${ }^{4}$ Mitchell Institute, Victoria University, Melbourne, Victoria, Australia

Contributors $\mathrm{BdC}$ designed the protocol for the study. KM wrote the first draft and revised the manuscript. MdC and JDC contributed to the writing of the protocol and revised the manuscript. All authors read and approved the manuscript.

Funding This work was supported by the Royal Australasian College of Physicians Carnosine supplement (CarnoPureTM) is received from Flamma S.p.A, Italy. There are no award or grant numbers for the funds or support received. KM is a recipient of the Research Training Programme (RTP) scholarship provided by Monash University. BdC is supported by Fellows Career Development Fellowship from the the Royal Australasian College of Physicians.

Disclaimer These funding bodies have no role in the design of the study and collection, analysis, and interpretation of data and writing of the manuscript.

Competing interests None declared.

Patient and public involvement Patients and/or the public were not involved in the design, or conduct, or reporting, or dissemination plans of this research.

Patient consent for publication Not required.

Provenance and peer review Not commissioned; externally peer reviewed.

Open access This is an open access article distributed in accordance with the Creative Commons Attribution Non Commercial (CC BY-NC 4.0) license, which permits others to distribute, remix, adapt, build upon this work non-commercially, and license their derivative works on different terms, provided the original work is properly cited, appropriate credit is given, any changes made indicated, and the use is non-commercial. See: http://creativecommons.org/licenses/by-nc/4.0/.

\section{ORCID iD}

Barbora de Courten http://orcid.org/0000-0001-8760-2511

\section{REFERENCES}

1 WHO. Obesity and overweight. Geneva: World Health Organization, 2020.

2 AlHW. Impact of overweight and obesity as a risk factor for chronic conditions: Australian burden of disease study. Canberra: Australian Institute of Health and Welfare, 2017.

3 Pi-Sunyer X. The medical risks of obesity. Postgrad Med 2009;121:21-33.

4 Pereira-Lancha LO, Campos-Ferraz PL, Lancha AH. Obesity: considerations about etiology, metabolism, and the use of experimental models. Diabetes Metab Syndr Obes 2012;5:75-87.

5 Weinsier RL, Hunter GR, Heini AF, et al. The etiology of obesity: relative contribution of metabolic factors, diet, and physical activity. Am J Med 1998;105:145-50.

6 Fernández-Sánchez A, Madrigal-Santillán E, Bautista M, et al. Inflammation, oxidative stress, and obesity. Int J Mol Sci 2011:12:3117-32.

7 Abe H. Role of histidine-related compounds as intracellular proton buffering constituents in vertebrate muscle. Biochemistry 2000;65:757-65.

8 Sale C, Artioli GG, Gualano B, et al. Carnosine: from exercise performance to health. Amino Acids 2013;44:1477-91.

9 Hipkiss AR, Michaelis J, Syrris P. Non-enzymatic glycosylation of the dipeptide I -carnosine, a potential anti-protein-cross-linking agent. FEBS Lett 1995;371:81-5.

10 Lee Y-ti, Hsu C-chin, Lin M-hsiao, et al. Histidine and carnosine delay diabetic deterioration in mice and protect human low density lipoprotein against oxidation and glycation. Eur J Pharmacol 2005;513:145-50.

11 Nagai K, Tanida M, Niijima A, et al. Role of L-carnosine in the control of blood glucose, blood pressure, thermogenesis, and lipolysis by autonomic nerves in rats: involvement of the circadian clock and histamine. Amino Acids 2012;43:97-109.

12 Pavlov AR, Revina AA, Dupin AM, et al. The mechanism of interaction of carnosine with superoxide radicals in water solutions. Biochim Biophys Acta 1993;1157:304-12.

13 Aydın AF, Küskü-Kiraz Z, Doğru-Abbasoğlu S, et al. Effect of carnosine against thioacetamide-induced liver cirrhosis in rat. Peptides 2010;31:67-71.

14 Forsberg EA, Botusan IR, Wang J, et al. Carnosine decreases IGFBP1 production in $\mathrm{db} / \mathrm{db}$ mice through suppression of HIF-1. $J$ Endocrinol 2015;225:159-67.

15 Mong M-chin, Chao C-yi, Yin M-chin. Histidine and carnosine alleviated hepatic steatosis in mice consumed high saturated fat diet. Eur J Pharmacol 2011;653:82-8.
16 Sauerhofer S, Yuan G, Braun GS, et al. L-carnosine, a substrate of carnosinase-1, influences glucose metabolism. Diabetes 2007;56:2425-32.

17 Yan S-L, Wu S-T, Yin M-C, et al. Protective effects from carnosine and histidine on acetaminophen-induced liver injury. J Food Sci 2009;74:H259-65.

18 Yan H, Harding JJ. Carnosine protects against the inactivation of esterase induced by glycation and a steroid. Biochim Biophys Acta 2005;1741:120-6.

19 Brown BE, Kim CHJ, Torpy FR, et al. Supplementation with carnosine decreases plasma triglycerides and modulates atherosclerotic plaque composition in diabetic apo E-/- mice. Atherosclerosis 2014;232:403-9.

20 Schön M, Mousa A, Berk M, et al. The potential of carnosine in Brain-Related disorders: a comprehensive review of current evidence. Nutrients 2019;11:1196.

21 de Courten B, Jakubova M, de Courten MPJ, et al. Effects of carnosine supplementation on glucose metabolism: pilot clinical trial. Obesity 2016;24:1027-34.

22 Houjeghani S, Kheirouri S, Faraji E, et al. L -carnosine supplementation attenuated fasting glucose, triglycerides, advanced glycation end products, and tumor necrosis factor- $\alpha$ levels in patients with type 2 diabetes: a double-blind placebo-controlled randomized clinical trial. Nutrition Research 2018;49:96-106.

23 Liu Y, Cotillard A, Vatier C, et al. A dietary supplement containing cinnamon, chromium and carnosine decreases fasting plasma glucose and increases lean mass in overweight or obese PreDiabetic subjects: a randomized, placebo-controlled trial. PLoS One 2015;10:e0138646.

24 Menon K, Marquina C, Hoj P, et al. Carnosine and histidinecontaining dipeptides improve dyslipidemia: a systematic review and meta-analysis of randomized controlled trials. Nutr Rev 2020;78:939-51.

25 Menon K, Marquina C, Liew D, et al. Histidine-containing dipeptides reduce central obesity and improve glycaemic outcomes: a systematic review and meta-analysis of randomized controlled trials. Obesity Reviews 2020;21:e12975.

26 Lombardi C, Carubelli V, Lazzarini V, et al. Effects of oral administration of orodispersible levo-carnosine on quality of life and exercise performance in patients with chronic heart failure. Nutrition 2015:31:72-8.

27 Szcześniak D, Budzeń S, Kopeć W, et al. Anserine and carnosine supplementation in the elderly: effects on cognitive functioning and physical capacity. Arch Gerontol Geriatr 2014;59:485-90.

28 Chan A-W, Tetzlaff JM, Gøtzsche PC, et al. SPIRIT 2013 explanation and elaboration: guidance for protocols of clinical trials. BMJ 2013;346:e7586

29 JG Y, Javorschi S, Hevener AL, et al. The effect of thiazolidinediones on plasma adiponectin levels in normal, obese, and type 2 diabetic subjects. Diabetes 2002;51:2968-74.

30 Knowler WC, Hamman RF, Edelstein SL, et al. Prevention of type 2 diabetes with troglitazone in the diabetes prevention program. Diabetes 2005;54:1150-6.

31 KKG L, DeMets DL. Discrete sequential boundaries for clinical trials. Biometrika 1983;70:659-63.

32 Svendsen OL, Haarbo J, Hassager C, et al. Accuracy of measurements of body composition by dual-energy X-ray absorptiometry in vivo. Am J Clin Nutr 1993;57:605-8.

33 Engelke K, Adams JE, Armbrecht G, et al. Clinical use of quantitative computed tomography and peripheral quantitative computed tomography in the management of osteoporosis in adults: the 2007 ISCD official positions. J Clin Densitom 2008;11:123-62.

34 Egerházi A, Berecz R, Bartók E, et al. Automated neuropsychological test battery (Cantab) in mild cognitive impairment and in Alzheimer's disease. Prog Neuropsychopharmacol Biol Psychiatry 2007;31:746-51.

35 Afdhal NH. Fibroscan (transient elastography) for the measurement of liver fibrosis. Gastroenterol Hepatol 2012;8:605-7.

36 Baye E, Ukropec J, de Courten MPJ, et al. Effect of carnosine supplementation on the plasma lipidome in overweight and obese adults: a pilot randomised controlled trial. Sci Rep 2017;7:17458.

37 Martin CA, Cameron JD, Chen SS, et al. Two hour glucose post loading: a biomarker of cardiovascular risk in isolated clinic hypertension. J Hypertens 2011;29:749-57.

38 Zoungas S, Cameron JD, Kerr PG, et al. Association of carotid intima-medial thickness and indices of arterial stiffness with cardiovascular disease outcomes in CKD. Am J Kidney Dis 2007:50:622-30.

39 Cheng K, Cameron JD, Tung M, et al. Association of left ventricular motion and central augmentation index in healthy young men. J Hypertens 2012;30:2395-402. 
40 Everaert I, Taes Y, De Heer E, et al. Low plasma carnosinase activity promotes carnosinemia after carnosine ingestion in humans. Am J Physiol Renal Physiol 2012;302:F1537-44.

41 Aldini G, Facino RM, Beretta G, et al. Carnosine and related dipeptides as quenchers of reactive carbonyl species: from structura studies to therapeutic perspectives. BioFactors 2005;24:77-87.

42 Forbes JM, Sourris KC, de Courten MPJ, et al. Advanced glycation end products (AGEs) are cross-sectionally associated with insulin secretion in healthy subjects. Amino Acids 2014;46:321-6.

43 Fenech M. Cytokinesis-block micronucleus cytome assay. Nat Protoc 2007;2:1084-104.

44 Thomas $\mathrm{P}$, Holland N, Bolognesi $\mathrm{C}$, et al. Buccal micronucleus cytome assay. Nat Protoc 2009;4:825-37.

45 EuroQol - a new facility for the measurement of health-related quality of life. Health Policy 1990;16:199-208.

46 Kroenke K, Strine TW, Spitzer RL, et al. The PHQ-8 as a measure of current depression in the general population. J Affect Disord 2009;114:163-73.

47 Craig CL, Marshall AL, Sjöström M, et al. International physical activity questionnaire: 12 -country reliability and validity. Med Sci Sports Exerc 2003;35:1381-95.

48 Buysse DJ, Reynolds CF, Monk TH, et al. The Pittsburgh sleep quality index: a new instrument for psychiatric practice and research. Psychiatry Res 1989;28:193-213.

49 Hoddes E, Zarcone V, Smythe H, et al. Quantification of sleepiness: a new approach. Psychophysiol 1973;10:431-6.

50 Monk TH, Reynolds CF, Kupfer DJ, et al. The Pittsburgh sleep diary. J Sleep Res 1994;3:111-20.

51 Aldini G, Orioli M, Rossoni G, et al. The carbonyl scavenger carnosine ameliorates dyslipidaemia and renal function in Zucker obese rats. J Cell Mol Med 2011;15:1339-54.

52 Yay A, Akkuș D, Yapıslar H, et al. Antioxidant effect of carnosine treatment on renal oxidative stress in streptozotocin-induced diabetic rats. Biotechnic \& Histochemistry 2014;89:552-7.

53 Albrecht T, Schilperoort M, Zhang S, et al. Carnosine attenuates the development of both type 2 diabetes and diabetic nephropathy in BTBR ob/ob mice. Sci Rep 2017;7:44492.

54 Barski OA, Xie Z, Baba SP, et al. Dietary carnosine prevents early atherosclerotic lesion formation in apolipoprotein E-Null mice. Arterioscler Thromb Vasc Biol 2013;33:1162-70.

55 Bao Y, Gao C, Hao W, et al. Effects of dietary L-carnosine and alpha-lipoic acid on growth performance, blood thyroid hormones and lipid profiles in finishing pigs. Asian-Australas J Anim Sci 2015;28:1465-70.

56 Rajanikant GK, Zemke D, Senut M-C, et al. Carnosine is neuroprotective against permanent focal cerebral ischemia in mice. Stroke 2007;38:3023-31.

57 Dobrota D, Fedorova T, Stvolinsky S, et al. Carnosine protects the brain of rats and Mongolian gerbils against ischemic injury: afterstroke-effect. Neurochem Res 2005;30:1283-8.

58 Fouad AA, El-Rehany MA-A, Maghraby HK. The hepatoprotective effect of carnosine against ischemia/reperfusion liver injury in rats. Eur J Pharmacol 2007;572:61-8.
59 Fujii T, Takaoka M, Muraoka T, et al. Preventive effect of L-carnosine on ischemia/reperfusion-induced acute renal failure in rats. Eur $J$ Pharmacol 2003;474:261-7.

60 Derosa G, D'Angelo A, Romano D, et al. A clinical trial about a food supplement containing $\alpha$-lipoic acid on oxidative stress markers in type 2 diabetic patients. Int J Mol Sci 2016;17:1802.

61 Elbarbary NS, Ismail EAR, El-Naggar AR, et al. The effect of 12 weeks carnosine supplementation on renal functional integrity and oxidative stress in pediatric patients with diabetic nephropathy: a randomized placebo-controlled trial. Pediatr Diabetes 2018;19:470-7

62 Corona C, Frazzini V, Silvestri E, et al. Effects of dietary supplementation of carnosine on mitochondrial dysfunction, amyloid pathology, and cognitive deficits in 3xTg-AD mice. PLoS One 2011;6:e17971.

$63 \mathrm{Ma} \mathrm{J}$, Xiong J-Y, Hou W-W, et al. Protective effect of carnosine on subcortical ischemic vascular dementia in mice. CNS Neurosci Ther 2012;18:745-53.

64 Hoffman JR, Landau G, Stout JR, et al. $\beta$-Alanine ingestion increases muscle carnosine content and combat specific performance in soldiers. Amino Acids 2015;47:627-36.

65 Boldyrev A, Fedorova T, Stepanova M, et al. Carnisone Increases Efficiency of DOPA Therapy of Parkinson's Disease: A Pilot Study. Rejuvenation Res 2008;11:821-7.

66 Solis MY, Cooper S, Hobson RM, et al. Effects of beta-alanine supplementation on brain homocarnosine/carnosine signal and cognitive function: an exploratory study. PLoS One 2015;10:e0123857.

67 Naderali EK, Ratcliffe SH, Dale MC, Obesity DMC. Review: Obesity and Alzheimer's Disease: A Link Between Body Weight and Cognitive Function in Old Age. Am J Alzheimers Dis Other Demen 2009;24:445-9.

68 Feng RN, Niu YC, Sun XW, et al. Histidine supplementation improves insulin resistance through suppressed inflammation in obese women with the metabolic syndrome: a randomised controlled trial. Diabetologia 2013;56:985-94.

69 Hipkiss AR, Cartwright SP, Bromley C, et al. Carnosine: can understanding its actions on energy metabolism and protein homeostasis inform its therapeutic potential? Chem Cent J 2013;7:38.

70 Guo X, Li H, Xu H, et al. Glycolysis in the control of blood glucose homeostasis. Acta Pharm Sin B 2012;2:358-67.

71 Coughlan MT, Yap FYT, Tong DCK, et al. Advanced glycation end products are direct modulators of $\beta$-cell function. Diabetes 2011;60:2523-32.

72 Lim M, Park L, Shin G, et al. Induction of apoptosis of $\beta$ cells of the pancreas by advanced glycation end-products, important mediators of chronic complications of diabetes mellitus. Ann N Y Acad Sci 2008;1150:311-5.

73 Herculano B, Tamura M, Ohba A, et al. $\beta$-alanyl-L-histidine rescues cognitive deficits caused by feeding a high fat diet in a transgenic mouse model of Alzheimer's disease. J Alzheimers Dis 2013;33:983-97. 